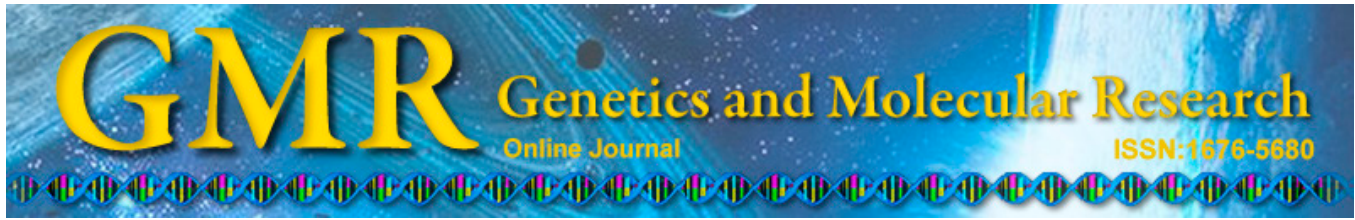

\title{
PI3K-AKT pathway polymerase chain reaction (PCR) array analysis of epilepsy induced by type II focal cortical dysplasia
}

Y.X. Lin ${ }^{1 *}$, K. Lin ${ }^{2 *}$, X.X. Liu ${ }^{3}$, D.Z. Kang ${ }^{1}$, Z.X. Ye ${ }^{1}$, X.F. Wang ${ }^{4}$, S.F. Zheng ${ }^{1}$, L.H. Yu ${ }^{1}$ and Z.Y. Lin ${ }^{1}$

${ }^{1}$ Department of Neurosurgery,

The First Affiliated Hospital of Fujian Medical University, Fuzhou, China

${ }^{2}$ Department of Neurosurgery, Fujian Provincial Hospital, Fuzhou, China

${ }^{3}$ Ultrasound Department,

The First Affiliated Hospital of Fujian Medical University, Fuzhou, China

${ }^{4}$ Department of Pathology,

The First Affiliated Hospital of Fujian Medical University, Fuzhou, China

*These authors contributed equally to this study.

Corresponding author: Y.X. Lin

E-mail: yuanxianglincn@163.com

Genet. Mol. Res. 14 (3): 9994-10000 (2015)

Received January 14, 2015

Accepted June 25, 2015

Published August 21, 2015

DOI http://dx.doi.org/10.4238/2015.August.21.5

\begin{abstract}
The aims of this study were to observe the differential expression of PI3K-AKT pathway-related genes in seizure-inducing brain lesions in type II focal cortical dysplasia, and to explore the relationship between gene expression and histological changes in dysplastic foci and their epileptogenic mechanism. Typical lesions in brain tissue from three patients with epilepsy induced by type II focal cortical dysplasia were selected for analysis, along with normal brain tissue from two control group individuals. Following quantitative expression analysis using the RT2 Profiler ${ }^{\mathrm{TM}}$ PI3K-AKT PCR Array, differential expression of the pathway related genes was detected in the focal brain tissue lesions, and gene function queries were performed. Compared with the control group, thirteen related genes appeared to exhibit marked differences in expression in epileptic lesions from
\end{abstract}


patients with type II focal cortical dysplasia; those genes were found to be involved in regulation of cell size, morphology, adhesion, migration, and apoptosis, and in immunity, inflammation, and many other domains. The differential expression of multiple genes in the PI3K-AKT signaling pathway in type II focal cortical dysplasia may be an important molecular mechanism underlying histological changes and recurrent seizures.

Key words: Cortical dysplasia; Epilepsy; Gene chip; PI3K-AKT signaling pathway

\section{INTRODUCTION}

There have been many studies demonstrating that the abnormal activation of the mammalian target of rapamycin (mTOR) protein in type II focal cortical dysplasia (FCD) lesions may be important in its pathogenesis (Miyata et al., 2004; Ljungberg et al., 2006; Schick et al., 2007; Orlova et al., 2010a; Wong, 2013). The mechanism of abnormal mTOR activation is not, however, well understood, although it may be related to somatic mutations in neuroglial stem cells during embryonic development (Crino, 2007). Furthermore, the mechanism of epileptogenesis consequent to abnormal activation of mTOR is also unclear. Currently, the determination of abnormal mTOR activation in type II FCD lesions is based on the detection of increased levels of phosphorylated forms of several key proteins of the phosphatidylinositol 3-kinase (PI3K)-AKT-mTOR pathway (Miyata et al., 2004; Ljungberg et al., 2006; Schick et al., 2007; Orlova et al., 2010a) in resected tissue specimens. To date, however, changes in PI3K-AKT-mTOR pathway protein RNA levels have not been reported in the literature. To address this deficit, we used the PI3K-AKT Signaling PCR Array functional classification microarray for simultaneous detection of expression levels of 84 genes in the signaling pathway. This profiling allowed us to achieve a comprehensive understanding of the signaling pathway-related protein expression changes in type II FCD lesions at the RNA level, in order to further confirm and complement the published experimental data.

\section{MATERIAL AND METHODS}

\section{Patient specimens}

Specimens were collected from patients undergoing surgery for refractory epilepsy in the Department of Neurosurgery of the First Affiliated Hospital of Fujian Medical University. Samples from patients confirmed to have typical type II FCD pathological changes in the cerebral cortex by postoperative pathology were taken as the experimental group $(\mathrm{N}=3)$; and resected cerebral cortex samples, that demonstrated no obvious lesions upon HE staining, and harvested from patients undergoing obligatory surgery for intraventricular neurocytoma, were taken as the control group $(\mathrm{N}=2)$. All specimens were preserved in $-196^{\circ} \mathrm{C}$ liquid nitrogen within $10 \mathrm{~min}$ following resection. This study was conducted in accordance with the Declaration of Helsinki, and with approval from the Ethics Committee of Fujian Medical University. Written informed consent was obtained from all participants.

\section{Polymerase chain reaction $(\mathrm{PCR})$}

Specimens were submitted to the Shanghai Kangcheng Biological Engineering Tech- 
nology Co. Ltd. for microarray analysis. The experimental steps were as follows: in accordance with manufacturer protocol, total cellular RNA was extracted following tissue homogenization (TRIZOL; Invitrogen, Carlsbad, CA, USA). RNA was purified according to the RNeasy ${ }^{\circledR}$ MinElute $^{\mathrm{TM}}$ Purification Kit (Qiagen, Dusseldorf, Germany) manufacturer protocol; RNA concentration and purity was determined by UV spectrophotometry; and its integrity was ascertained by denaturing agarose gel electrophoresis. RNA was reverse transcribed to cDNA using the SuperScript III Transcriptase kit (Invitrogen). Real-time quantitative PCR was performed using the 96-Well RT2 Profiler ${ }^{\mathrm{TM}}$ PCR Array (Qiagen) according to the manufacturer protocol as follows: after appropriate dilution, the cDNA template was added to the real-time quantitative reaction mixture, and equal amounts of reaction liquid were added to each well of the PCR array, containing gene specific primers. Conditions for the real-time quantitative PCR reaction were as follows: denaturing at $95^{\circ} \mathrm{C}$ for $10 \mathrm{~min}, 40$ amplification cycles of annealing at $95^{\circ} \mathrm{C}$ for 10 $\mathrm{s}$, and extension at $60^{\circ} \mathrm{C}$ for $1 \mathrm{~min}$, followed by acquisition of fluorescence signal. Data analysis is based on the $\Delta \Delta \mathrm{Ct}$ method with normalization raw data to housekeeping genes by SKBET.

\section{RESULTS}

\section{Concentration and purity of specimen RNA}

The concentration of extracted RNA ranged from $375.89 \mathrm{ng} / \mu \mathrm{L}$ to $634.78 \mathrm{ng} / \mu \mathrm{L}$, with OD260/OD280 ratios ranging from 1.8 to 2.1 , in accordance with experimental requirements (Table 1).

Table 1. Source, concentration, and purity of RNA specimens.
\begin{tabular}{lcrccc}
\hline Specimen serial number & Sample group & OD260 & OD280 & OD260/OD280 & Concentration (ng/ $\mu \mathrm{L})$ \\
\hline 1 & FCD II & 9.397 & 4.889 & 1.92 & 375.89 \\
2 & FCD II & 13.045 & 6.631 & 1.97 & 521.81 \\
3 & FCD II & 15.869 & 7.771 & 2.04 & 634.78 \\
4 & Control & 9.542 & 4.997 & 1.91 & 381.67 \\
5 & Control & 9.828 & 5.142 & 1.91 & 393.10 \\
\hline
\end{tabular}

FCD II = focal cortical dysplasia type II.

\section{Identification of specimen RNA integrity}

Following denaturing agarose gel electrophoresis, thick bright bands of $28 \mathrm{~s}$ and $18 \mathrm{~s}$ ribosomal RNA were observed for each RNA sample (Figure 1), indicating that the specimen RNA integrity was acceptable.

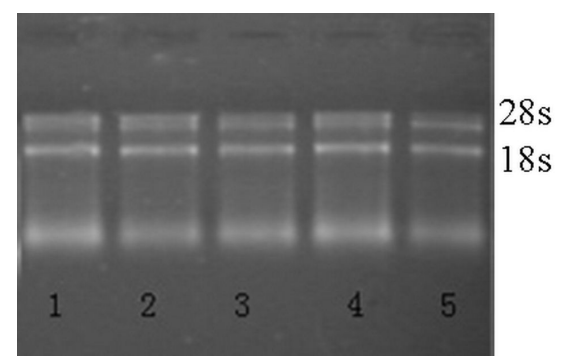

Figure 1. Electrophoretogram of total RNAs extracted from brain tissue. Lanes 1-3 contain type II FCD lesion RNA; lanes 4 and 5 contain RNA from control group samples. 


\section{PI3K-AKT signaling pathway related gene expression}

The mRNA expression levels of 84 genes related to the PI3K-AKT signaling pathway were detected by quantitative PCR array. Compared with the control group, thirteen genes demonstrated a greater than two-fold change in expression in type II FCD lesions, including twelve upregulated genes: Bruton agammaglobulinemia tyrosine kinase $(B T K)$; CD14; eukaryotic translation initiation factor 4E binding protein 1 (EIF4BP1); Fas ligand $(F A S L G)$; Finkel-Biskis-Jinkins (FBJ) murine osteosarcoma viral oncogene homolog $(F O S)$; heat shock 27-kDa protein 1 (HSPB1); integrin beta 1 (ITGB1); jun proto-oncogene $(J U N)$; myeloid differentiation primary response gene 88 (MYD88); phosphoinositide-3-kinase, catalytic, gamma polypeptide (PIK3CG); ribosomal protein S6 kinase, 90-kDa polypeptide 1 (PRS6KA1); T-cell leukemia/lymphoma 1A (TCL1A); and a single downregulated gene, P21 protein (Cdc42/Rac)-activated kinase 1 (PAK1). In contrast, no substantial changes in mRNA expression were observed for proteins previously reported in the literature to exhibit significantly increased protein phosphorylation in type II FCD lesions: v-akt murine thymoma viral oncogene homolog 1 (AKT1), AKT2, AKT3, mTOR, pyruvate dehydrogenase kinase, isozyme 1 (PDK1), PDK2, and ribosomal protein S6 kinase, 70-kDa polypeptide 1 (RPS6KB1). Substantial changes were also not identified in the mRNAs of several key proteins associated with type II FCD including tuberous sclerosis 1 (TSC1), phosphatase and tensin homolog (PTEN), and tuberous sclerosis 2 (TSC2) (Table 2).

Table 2. Relative expression in type II FCD of genes involved in the PI3K-AKT signaling pathway.

\begin{tabular}{|c|c|c|c|c|c|}
\hline Gene name & Fold up- or downregulated & Gene name & Fold up- or downregulated & Gene name & Fold up- or downregulated \\
\hline ADAR & -1.29 & GRB2 & 0.59 & PIK3CG & 2.07 \\
\hline AKT1 & -1.12 & GSK3B & 0.89 & PIK3R1 & 0.49 \\
\hline AKT2 & 1.05 & HRAS & 0.63 & PIK3R2 & 0.94 \\
\hline AKT3 & -1.65 & HSPB1 & 1.29 & PRKCA & 1.44 \\
\hline APC & -1.33 & IGF1 & 2.92 & PRKCB & 0.38 \\
\hline $\mathrm{BAD}$ & -1.37 & IGF1R & 0.84 & PRKCZ & 0.67 \\
\hline BTK & 3.49 & ILK & 0.95 & PTEN & 1.06 \\
\hline CASP9 & 1.04 & IRAK1 & 0.93 & PTK2 & 1.27 \\
\hline CCND1 & 1.28 & IRS1 & 0.91 & PTPN11 & 0.82 \\
\hline CD14 & 8.35 & ITGB1 & 0.57 & $\mathrm{RAC1}$ & 0.84 \\
\hline $\mathrm{CDC} 42$ & -1.11 & JUN & 2.02 & RAF1 & 0.92 \\
\hline CDKN1B & 1.04 & MAP2K1 & 2.39 & RASA1 & 0.73 \\
\hline CHUK & 1.11 & MAPK1 & 0.74 & RBL2 & 1.08 \\
\hline CSNK2A1 & 1.10 & MAPK14 & 0.85 & RHEB & 0.99 \\
\hline CTNNB1 & -1.13 & MAPK3 & 0.62 & RHOA & 1.50 \\
\hline EIF2AK2 & -1.19 & MAPK8 & 0.75 & RPS6KA1 & 2.21 \\
\hline EIF4B & -1.18 & MTCP1 & 0.62 & RPS6KB1 & 0.87 \\
\hline EIF4E & -1.18 & MYD88 & 0.97 & SHC1 & 1.25 \\
\hline EIF4EBP1 & 2.53 & NFKB1 & 3.21 & SOS1 & 1.14 \\
\hline EIF4G1 & -1.09 & NFKBIA & 1.03 & SRF & 1.02 \\
\hline ELK1 & 1.01 & PABPC1 & 1.63 & TCL1A & 6.29 \\
\hline FASLG & 3.27 & PAK1 & 1.61 & TIRAP & 0.85 \\
\hline FKBP1A & 1.01 & PDGFRA & 0.30 & TLR4 & 1.26 \\
\hline FOS & 23.61 & PDK1 & 1.13 & TOLLIP & 0.60 \\
\hline FOXO1 & 1.96 & PDK2 & 0.75 & TSC1 & 0.79 \\
\hline FOXO3 & -1.12 & PDPK1 & 0.62 & TSC2 & 0.96 \\
\hline MTOR & -1.37 & PIK3CA & 0.53 & WASL & 0.58 \\
\hline GJA1 & 1.34 & PIK3CG & 0.78 & YWHAH & 0.56 \\
\hline
\end{tabular}

FCD $=$ focal cortical dysplasia. 


\section{DISCUSSION}

The mammalian target of rapamycin (mTOR) is a highly conserved serine/threonine protein kinase, and much evidence has accumulated indicating that the mTOR signaling pathway plays an important role in the pathogenesis of epilepsy (Meng et al., 2013; Yasin et al., 2013; Lee et al., 2014; Scheffer et al., 2014). TSC is an autosomal dominant hereditary disease denoted by epilepsy relating to aberrant activation of mTOR caused by TSC1/TSC 2 gene mutation. Experiments have confirmed that inhibition of the mTOR pathway can inhibit epileptic seizures in patients with TSC and in animal models, and can even reverse the pathological changes (Magri et al., 2013; Cardamone et al., 2014). Polyhydramnios, megalencephaly, and symptomatic epilepsy syndrome (PMSE) is a rare human autosomal recessive hereditary disease caused by homozygous deletion of the STE20-related kinase adaptor $\alpha$ (STRADA) gene, leading to abnormal mTOR activation, with patients exhibiting large skull, craniofacial deformities, myasthenia gravis, cognitive impairment, and refractory epilepsy (Orlova et al., 2010b); inhibition of mTOR can stop the epileptic seizure in animal models of this disorder (Parker et al., 2013). The similarities in morphological structures between FCD II, and TSC and PMSE patients, combined with our previous experimental results along with related publications, have demonstrated that type II FCD lesions have differential, elevated levels of phosphorylated forms of key proteins such as p-PDK1 (phosphorylated at Ser241), p-AKT (Thr308), p-AKT (Ser473), p-mTOR (Ser2448), p-P70S6K (Thr229 and Thr389), p-P70S6K, p-4E-BP1, p-elF4G, p-S6, and other key proteins in the PI3K-AKT-mTOR pathway; which in turn confirmed the abnormal activation of the PI3K-AKT-mTOR signaling pathway in type II FCD lesions (Miyata et al., 2004; Ljungberg et al., 2006; Schick et al., 2007; Orlova et al., 2010a). Because, unlike TSC and PMSE, no familial aggregation has been observed to date for patients with type II FCD, many scholars have speculated that type II FCD might be a disorder arising from somatic gene mutation (Crino, 2007; Lim and Crino, 2013). We hypothesized that a gene mutation in neuroglial stem cells in the early embryonic period might lead to aberrant activation of PI3K-AKT-mTOR, thereby causing the formation of type II FCD lesions. Due to the multiple components involved in the PI3K-AKT signaling pathway, at present, we are unable to simultaneously study all of the components involved in the signal transduction pathway at the level of the protein. At the same time, because of co-regulatory relationships between components involved in the signal transduction pathway when a protein is found to be abnormally activated, we are unable to determine whether this arises from abnormal expression of the protein itself, or it is due to abnormal expression of an upstream molecule leading to abnormal activation. The PI3K-AKT Signaling PCR Array simultaneously detects expression of 84 genes in the signaling pathway, to achieve a comprehensive understanding of the molecular profile. Our experimental results showed that the mRNA expression levels of AKT1, AKT2, AKT3, mTOR, PDK1, PDK2, and RPS6KB1 in type II FCD lesions were not higher than those in the control tissue, suggesting that the increased expression of phosphorylated forms of these proteins in these lesions might not be a consequence of high expression of the protein itself. We did, however, identify an increase in TCL1A expression, which can promote phosphorylation of AKT and the downstream mTOR and P70S6K proteins, leading to their activation; thus potentially explaining the documented increased expression of phosphorylated forms of these key proteins of the PI3K-AKT-mTOR pathway in type II FCD lesions. Therefore, changes in TCL1A protein levels in type II FCD lesions, along with cellular distribution, and abnormal expression mechanisms are worth further study. Finally, consistent 
with the experimental results of Schick et al. (2006), we found no change in PTEN expression, indicating that the abnormal activation of AKT in type II FCD lesions might not be associated with PTEN regulatory mutations.

Our experimental results provide the first demonstration that the expression of the inflammation and immune related factors BTK, CD14, and MYD 88 were increased in FCD II lesions. According to experimental result, mTOR signaling pathway plays an important role in immune and inflammatory reactions (Weichhart and Säemann, 2009), suggesting that the elevated expression of inflammatory cytokines may be the result of abnormal activation of mTOR. The human papilloma virus type 16 (HPV16) E6 oncoprotein can activate the mTOR signaling pathway at different stages and different levels (Lu et al., 2004). HPV DNA, mRNA, and related proteins have been found in type II FCD surgical specimens (Chen et al., 2012). On the other hand, as congenital cytomegalovirus infection can cause focal pachygyria, some authors have suggested that type II FCD may be result of activation of mTOR caused by early intrauterine infection (Lim and Crino, 2013); therefore the increased expression of the inflammation and immune related factors might be the result of intrauterine infection. Of course, as reported in the literature, these immune and inflammation factors themselves might be involved in the underlying epileptogenic mechanisms of type II FCD (Iyer et al., 2010).

Much research in recent years has indicated that the occurrence of epilepsy is closely related to the disruption of a variety of cerebral apoptosis genes (Friedman, 2010). Our experiments found the increased expression of apoptosis related factors, such as FASLG and FOS, indicating that the abnormal activation of mTOR-mediated apoptosis may be involved in the epileptogenic mechanisms of type II FCD-related epilepsy, which is consistent with the experimental results of Iyer et al. (2014).

In addition, our experimental results showed the abnormal expression of some molecules relating to the histological changes of type II FCD. Our experimental results demonstrated increased expression of ITGB1, a negative regulator of collagen receptors leading to negative regulation of the adhesion and migration of cells, which is consistent with the histopathological finding of obvious structural disorder in type II FCD (Blümcke et al., 2011). Similarly, the RPS6KA1 protein is a ubiquitously expressed serine/threonine kinase, which not only plays an important role in glucose homeostasis, but also plays an important role in the regulation of cell size. PAK1 plays an important role in the regulation of cell morphology and mitosis. Our experimental results showed increased expression of RPS6KA1, and downregulated expression of $P A K 1$, which could partially explain the characteristic balloon cells and abnormal morphological changes of neurons in type II FCD lesions (Blümcke et al., 2011).

In conclusion, multiple genes in the PI3K-AKT signaling pathway appear to be differentially expressed in type II FCD lesions, and the differential expression of these genes supports abnormal activation of the signaling pathway in type II FCD lesions, and can explain the histological changes of type II FCD lesions and the epileptogenic mechanism to some extent. These results demonstrate that the pathogenic mechanism of PI3K-AKT signaling pathway in type II FCD lesions is worth further study.

\section{ACKNOWLEDGMENTS}

Research supported by the Natural Science Foundation of Fujian Province (\#2011J01164), the Fujian Province Key Clinical Subject Construction Project, and special 
fund for cultivating academic leaders of Fujian Medical University (\#JXK201301).

\section{REFERENCES}

Blümcke I, Thom M, Aronica E, Armstrong DD, et al. (2011). The clinicopathologic spectrum of focal cortical dysplasias: a consensus classification proposed by an ad hoc Task Force of the ILAE Diagnostic Methods Commission. Epilepsia 52: $158-174$.

Cardamone M, Flanagan D, Mowat D, Kennedy SE, et al. (2014). Mammalian target of rapamycin inhibitors for intractable epilepsy and subependymal giant cell astrocytomas in tuberous sclerosis complex. J. Pediatr. 64: 1195-1200.

Chen J, Tsai V, Parker WE, Aronica E, et al. (2012). Detection of human papillomavirus in human focal cortical dysplasia type IIB. Ann. Neurol. 72: 881-892.

Crino PB (2007). Focal brain malformations: a spectrum of disorders along the mTOR cascade. Novartis Found. Symp. 288: 260-272; Discussion 272-281.

Friedman WJ (2010). Proneurotrophins, seizures, and neuronal apoptosis. Neuroscientist 16: 244-252.

Iyer A, Zurolo E, Spliet WG, van Rijen PC, et al. (2010). Evaluation of the innate and adaptive immunity in type I and type II focal cortical dysplasias. Epilepsia 51: 1763-1773.

Iyer A, Prabowo A, Anink J, Spliet WG, et al. (2014). Cell injury and premature neurodegeneration in focal malformations of cortical development. Brain Pathol. 24: 1-17.

Lee JY, Park AK, Lee ES, Park WY, et al. (2014). miRNA expression analysis in cortical dysplasia: Regulation of mTOR and LIS1 pathway. Epilepsy Res. 108: 433-441.

Lim KC and Crino PB (2013). Focal malformations of cortical development: New vistas for molecular pathogenesis. Neuroscience 252: 262-276.

Ljungberg MC, Bhattacharjee MB, Lu Y, Armstrong DL, et al. (2006). Activation of mammalian target of rapamycin in cytomegalic neurons of human cortical dysplasia. Ann. Neurol. 60: 420-429.

Lu Z, Hu X, Li Y, Zheng L, et al. (2004). Human papillomavirus 16 E6 oncoprotein interferences with insulin signaling pathway by binding to tuberin. J. Biol. Chem. 279: 35664-35670.

Magri L, Cominelli M, Cambiaghi M, Cursi M, et al. (2013). Timing of mTOR activation affects tuberous sclerosis complex neuropathology in mouse models. Dis. Model Mech. 6: 1185-1197.

Meng XF, Yu JT, Song JH, Chi S, et al. (2013). Role of the mTOR signaling pathway in epilepsy. J. Neurol. Sci. 332: 4-15.

Miyata H, Chiang AC and Vinters HV (2004). Insulin signaling pathways in cortical dysplasia and TSC-tubers: tissue microarray analysis. Ann. Neurol. 56: 510-519.

Orlova KA, Parker WE, Heuer GG, Tsai V, et al. (2010a). STRADalpha deficiency results in aberrant mTORC1 signaling during corticogenesis in humans and mice. J. Clin. Invest. 120: 1591-1602.

Orlova KA, Tsai V, Baybis M, Heuer GG, et al. (2010b). Early progenitor cell marker expression distinguishes type II from type I focal cortical dysplasias. J. Neuropathol. Exp. Neurol. 69: 850-863.

Parker WE, Orlova KA, Parker WH, Birnbaum JF, et al. (2013). Rapamycin prevents seizures after depletion of STRADA in a rare neurodevelopmental disorder. Sci. Transl. Med. 5: 182ra153.

Scheffer IE, Heron SE, Regan BM, Mandelstam S, et al. (2014). Mutations in mTOR regulator DEPDC5 cause focal epilepsy with brain malformations. Ann. Neurol. 75: 782-787.

Schick V, Majores M, Engels G, Spitoni S, et al. (2006). Activation of Akt independent of PTEN and CTMP tumor-suppressor gene mutations in epilepsy-associated Taylor-type focal cortical dysplasias. Acta Neuropathol. 112: 715-725.

Schick V, Majores M, Engels G, Hartmann W, et al. (2007). Differential Pi3K-pathway activation in cortical tubers and focal cortical dysplasias with balloon cells. Brain Pathol. 17: 165-173.

Weichhart T and Säemann MD (2009). The multiple facets of mTOR in immunity. Trends Immunol. 30: 218-226.

Wong M (2013). Mammalian target of rapamycin (mTOR) activation in focal cortical dysplasia and related focal cortical malformations. Exp. Neurol. 244: 22-26.

Yasin SA, Ali AM, Tata M, Picker SR, et al. (2013). mTOR-dependent abnormalities in autophagy characterize human malformations of cortical development: evidence from focal cortical dysplasia and tuberous sclerosis. Acta Neuropathol. 126: 207-218. 